\title{
Preface: Oncogenesis: Special Focus on Pancreatic Cancer
}

Pancreatic cancer, most commonly known as pancreatic ductal adenocarcinoma, is the fourth leading cause of cancer deaths in the United States. It is particularly lethal because of the difficulty in detecting its early onset and its intrinsic resistance to therapy. Pancreatic cancer is generally silent until later stages, when the patient finally experiences nonspecific symptoms such as abdominal pain, weight loss, and jaundice. At this point, the cancer has usually progressed to a metastatic late stage. Although a combination of chemotherapy, radiation, and surgical intervention is the standard therapy, survival rates remain below $10 \%$.

Resistance to treatment is due to pancreatic cancer's dense stroma, as well as its Sonic Hedgehog, $\beta$-integrin, and Stat3 signaling activities, enhanced metastasis, and chemoresistance. Also, pancreatic tumors are composed of a densely packed extracellular matrix, which collapses blood vessels and decreases chemotherapy delivery to the tumor. These unique characteristics make pancreatic cancer aggressive and deadly.

In the current issue, we present nine review articles relevant to pancreatic cancer growth, metastasis, diagnosis, and treatment. The first review discusses current advances in immunotherapeutic strategies, including checkpoint inhibitors such as the FDAapproved pembrolizumab and T-cell inducers. The second review explores developments in molecular, cytogenetic, and translational applications, and shines light on the development of promising diagnostic and treatment strategies based on personalized precision medicine. The third review examines forensic strategies for detection, including DNA fingerprinting based on inheritance disease patterns. The fourth review focuses on nanoparticles in imaging and drug delivery. Nanoparticles have emerged as a promising tool for advancing precision medicine because of their nano scale and properties. In cancer therapeutics, they have expanded the arsenal of treatments in a variety of tumors and may thus enhance the cytotoxic activities of conventional or novel treatments in pancreatic cancer.

The fifth review discusses familial pancreatic cancer, examining various syndromes and genetic alterations associated with it and recommending screening strategies. The sixth review provides a thorough overview of current advances in therapeutic strategies, with an emphasis on genetic mutations, immune therapy, and targeted proteomic approaches. The seventh review explores the use of exosomes as both therapeutic targets and drug delivery vehicles, particularly engineered exosomes and exosome mimetics in prognosis, monitoring, and drug delivery. It also presents the latest updates on the exosome signature of pancreatic cancer and its role in tumor progression. The eighth review offers perspectives on computational biology in early detection. The ninth and final review explores pathways of resistance to gemcitabine, the current standard of treatment in pancreatic cancer.

This special issue of Critical Reviews in Oncogenesis offers a broad overview of recent developments in pancreatic cancer detection and treatment, exploring various facets of research in this field. A solid understanding of cytogenic, molecular, and transitional applications is critical for improving detection and treatment modalities. New applications of existing technologies are expected to revolutionize the treatment response. DNA fingerprinting technology and computational biology may improve detection, while nanoparticles, exosomes, and immunotherapeutics may enhance imaging and therapeutic strategies. The contributors to this special issue have reviewed and discussed all of these challenging topics. With further advances in innovative detection and therapy systems, we hope that the cancer's mortality can be reduced as it has been in other cancers.

\section{Guest Editor:}

Ganji Purnachandra Nagaraju, PhD, DSc, FAACC Winship Cancer Institute, Emory University Department of Hematology and Medical Oncology Atlanta, GA 